\title{
Specificity of the Point-of-Care Urine Strip Test for Schistosoma Circulating Cathodic Antigen (POC-CCA) Tested in Non-Endemic Pregnant Women and Young Children
}

\author{
Miriam Casacuberta-Partal, ${ }^{1 \star}$ Margreet Beenakker, ${ }^{1}$ Claudia J. de Dood, ${ }^{2}$ Pytsje T. Hoekstra, ${ }^{1}$ Lisa Kroon, ${ }^{1}$ Dieuwke Kornelis, \\ Paul Corstjens, ${ }^{2}$ Cornelis H. Hokke, ${ }^{1}$ Govert J. van Dam, ${ }^{1}$ Meta Roestenberg, ${ }^{1,3}$ and Lisette van Lieshout ${ }^{1}$ \\ ${ }^{1}$ Department of Parasitology, Leiden University Medical Centre, Leiden, The Netherlands; ${ }^{2}$ Department of Cell and Chemical Biology, Leiden \\ University Medical Center, Leiden, The Netherlands; ${ }^{3}$ Department of Infectious Diseases, Leiden University Medical Center, Leiden, The
} Netherlands

\begin{abstract}
The point-of-care urine based strip test for the detection of circulating cathodic antigen (POC-CCA) in schistosome infections is a frequently used tool for diagnosis and mapping of Schistosoma mansoni in school-aged children. Because of its ease of use, the test is increasingly applied to adults and preschool-aged children (PSAC), but its performance has not been specifically evaluated in these target groups. Recent observations have raised concerns about possible reduced specificity, in particular in pregnant women (PW) and PSAC. We thus explored specificity of the POCCCA urine strip test (Rapid Medical Diagnostics, Pretoria, South Africa) in a non-endemic, nonexposed population of 47 healthy nonpregnant adults (NPAs), 52 PW, and 58 PSAC. A total of 157 urines were tested with POC-CCA, of which five (10.6\%) NPAs, 17 (32.7\%) PW, and 27 (46.5\%) PSAC were positive. The highest scores were found in the youngest babies, with an infant of 9 months being the oldest positive case. On measuring $\mathrm{pH}$, it appeared that all POC-CCA strongly positive urines were acidic ( $\mathrm{pH}$ range 5-5.5), whereas addition of $\mathrm{pH}$-neutral buffer to a subsample reversed the false positivity. We conclude that the POC-CCA test has reduced specificity in PW and infants younger than 9 months, but that the false positivity might be eliminated by modifications in the buffers used in the test.
\end{abstract}

\section{INTRODUCTION}

Schistosomiasis is a parasitic infection caused by trematode worms of the genus Schistosoma. It is a neglected tropical disease, affecting mainly populations living in poverty without adequate sanitation. ${ }^{1}$ According to the $\mathrm{WHO}$, at least 290 million people required treatment for this infection in $2018 .^{2}$ In high-risk communities, the assessment of prevalence and intensity of infection is based on microscopic detection of parasite eggs in stool or urine samples, a readily available and highly specific diagnostic procedure which lacks sensitivity particularly when egg excretion is low. Microscopy might thus underestimate the true prevalence of infection. Hence, a urine-based point-of-care (POC) test for the detection of circulating cathodic antigen (CCA) is increasingly used since its commercial availability in 2005 , as it is a userfriendly, affordable, and highly sensitive and specific alternative for microscopy. ${ }^{3}$ The POC-CCA test has been particularly useful for mapping the distribution of intestinal schistosomiasis in sub-Saharan African regions. ${ }^{4-7}$ Recently, the test has been integrated into the WHO-recommended treatment strategy for controlling morbidity. 8,9

Circulating cathodic antigen is a glycan antigen derived from the gut of schistosome worms. It contains one or more carbohydrate chains consisting of repeating Lewis- $x$ (LeX) trisaccharides attached to an O-linked glycan core. ${ }^{10}$ The POC-CCA test uses a nitrocellulose strip in combination with a colloid gold conjugate of an anti-CCA monoclonal antibody that recognizes the carbohydrate chain. ${ }^{4}$ The CCA antigen binds to the antibody immobilized on the strip when urine containing the antigen flows on the strip. Although CCA is known to be excreted by all Schistosoma species, the POCCCA urine test has shown to be most sensitive for the

\footnotetext{
*Address correspondence to Miriam Casacuberta-Partal, Department of Parasitology, Leiden University Medical Centre, P.O. Box 9600, Leiden 2333 ZA, The Netherlands. E-mail: m.casacuberta_ partal@lumc.nl
}

diagnosis of intestinal African schistosomiasis. ${ }^{5}$ The intensity of the test line can even be read in a semiquantitative way and interpreted as a reflection of worm burden, and therefore be used for monitoring disease control. ${ }^{5,11}$ Several studies have demonstrated a rapid reduction of the test line intensity following treatment with the anti-Schistosoma drug praziquantel. $^{12-14}$

Although a large number of studies have successfully shown the POC-CCA urine test to be a valuable alternative to stool microscopy for the diagnosis of Schistosoma mansoni infections, the focus of these evaluation studies has been mainly on school-aged children and adults. ${ }^{5,6,15}$ There are limited data regarding the performance of the POC-CCA urine test in pregnant women (PW) and preschool-aged children (PSAC). This is because PW and PSAC have often been excluded in control programs based on mass drug administration. ${ }^{16,17}$

The performance of the POC-CCA test in PW and PSAC has been debated following incidental reports of possibly nonspecific test results. In a small survey performed in Chad found that of a group of 49 women aged 14 years and older, six females, of which three were pregnant, had a positive POCCCA urine test while being negative for all other diagnostic procedures used. ${ }^{18}$ The discussion was further ignited when two Swiss PW without any history of visiting a schistosomeendemic region were tested and one of them showed a positive outcome. ${ }^{18}$ Based on our own observations and on critical analysis of field collected data, we had the impression that particularly in children younger than 5 years, positive POC-CCA results could disproportionally often not be confirmed by microscopy. Because of the chemical structure of the immunoreactive CCA portion consisting of linear LeX repeats, it has been suggested that the anti-CCA monoclonal antibodies used in the POC-CCA strip test might cross-react with host-derived urinary glycans or glycoconjugate that might also contain multiple LeX motifs. ${ }^{10}$

To further explore the suggested reduced specificity of the POC-CCA urine test in PW and PSAC, we assessed the performance of the test in a non-endemic population, resident in 
the Netherlands, without any known exposure to a Schistosoma infection. In this study, urine samples of three categories of individuals were collected and tested: 1) nonpregnant adults (NPAs), 2) PW, 3) PSAC.

\section{MATERIALS AND METHODS}

Ethics statement. Ethics approval was obtained from the Ethics Committee of the Leiden University Medical Center in the Netherlands (P16.164, date of approval October 12, 2017). Participants were informed on the purpose and procedures of the study. Written informed consent was obtained from all adult participants and from the parents of the participating children.

Participant recruitment. To be included in the study, each participant had to be a resident of the Netherlands and had to provide one urine sample of at least $0.5 \mathrm{~mL}$. Those who had previous contact with open fresh water in schistosome endemic areas such as South America, Africa, Southeast Asia, or Corsica, and/or a history of schistosomiasis were excluded. Also excluded were those urines showing a positive bacterial culture following examination at the microbiology department or complaints of urinary infection. This is because urinary tract infections are already known to potentially interfere with the POC-CCA urine strip test (Rapid Medical Diagnostics Technical Brochure, Pretoria, South Africa). ${ }^{19}$

Urine was requested of 47 nonpregnant, healthy adults (NPAs) in the age range of 18-65 years. This group served as a control. Pregnant women $(N=52)$ were recruited via the outpatient clinic of the gynecology department of the Leiden University Medical Center (LUMC). Besides the request for a urine sample, the PW were questioned about their age and their pregnancy duration. Residual urines $(N=58)$ of children younger than 4 years were obtained via the Department of Clinical Chemistry of the LUMC.

All urine samples were anonymized and were tested blinded for age, gender, and study category with the POC-CCA urine strip test, as well as with an urine reagent strip and an upconverting reporter particle-based lateral flow strip assay for the detection of schistosome circulating anodic antigen (UCPLF CAA), as described in the following text.

Sample size calculation. Based on many years of experience using the POC-CCA urine strip assay in endemic and non-endemic settings, an overall diagnostic specificity of $95 \%$ was assumed. ${ }^{5}$ Allowing a drop of $20 \%$ of the specificity in the PW and PSAC urine, three groups of 50 participants were needed to conduct this study with $\alpha=0.05$ and $\beta=0.8$. $^{20}$

Study procedures. Point-of-care-circulating cathodic antigen testing. The POC-CCA test (batch number: 170522062, expiration date: May 2019) was performed according to the manufacturer's instructions (Rapid Medical Diagnostics). ${ }^{19}$ The cassettes were read 20 minutes after adding the urine sample by two blinded investigators who compared the intensity of the test line with the preprinted $10 \mathrm{G}$-scores as previously described to standardize the readings. ${ }^{11}$ Subsequently, G-scores were recoded into the more widely used visual categories (negative, trace, $1+, 2+$, and $3+$ ). Four urine reference samples ( $S$-series) with known different antigen concentration were used as standards to calibrate the Gscores with the used POC-CCA batch number. ${ }^{11}$

Up-converting reporter particle-based lateral flow strip assay for the detection of schistosome circulating anodic antigen. To confirm the negative schistosome infection status, urines were tested with the high sensitivity UCP-LF CAA assay as described before using a slightly modified UCAAhT417 protocol of the wet test format. ${ }^{21}$ Only urines of sufficient remaining volume $(>0.5 \mathrm{~mL})$ following the POC-CCA testing were included. In brief, $0.5 \mathrm{~mL}$ urine samples were pretreated with $12 \%$ trichloroacetic acid (TCA) to remove interfering proteins and to dissociate potential immune complexes as indicated in Corstjens et al. ${ }^{21}$ Following a centrifugation step, the supernatant resulting from the TCA extraction was reduced to a volume of approximately $20 \mu \mathrm{L}$ using a $0.5-\mathrm{mL}$ spin filter with a 10-kDa molecular weight cutoff (Amicon Ultra, Millipore Sigma, Merck Chemicals B.V, Amsterdam, The Netherlands) and further tested as described earlier. ${ }^{22}$

Urinalysis reagent strip. The presence of protein and the $\mathrm{pH}$ level were tested via the urine reagent strip test (Combur 7 Test $^{\circledR}$, Roche Diagnostics, Gmbh, Manheim, Germany). In brief, a strip was dipped for about one second in each urine sample. The readings were performed according to the manufacturer's instructions by paring the color of the reaction of the strip after a minute to the matching standard labeled on the bottle.

Statistical analyses. The test outputs are presented semiquantitatively for the visual scoring of the POC-CCA test. A POC-CCA test was considered positive when the test line was visually scored as $1+$ or above, and only when indicated within the analyses, the traces were included as positive. ${ }^{11}$ Specificity was estimated for each group using the relationship between the true disease class and the observed POC-CCA results, assuming that all participants have never been exposed to schistosomiasis, based on our exclusion criteria. Percentage of positive tests between PW and PSAC was compared with NPA using Fisher's exact test. Proportions, means, or, where appropriate, medians and interquartile range (IQR) were calculated. All $P$-values smaller than 0.05 were considered as significant.

\section{RESULTS}

A total of 157 individuals living in the Netherlands participated in this study by providing a urine sample (Figure 1). Of the 47 NPA urines, nine individuals (19\%) showed a positive

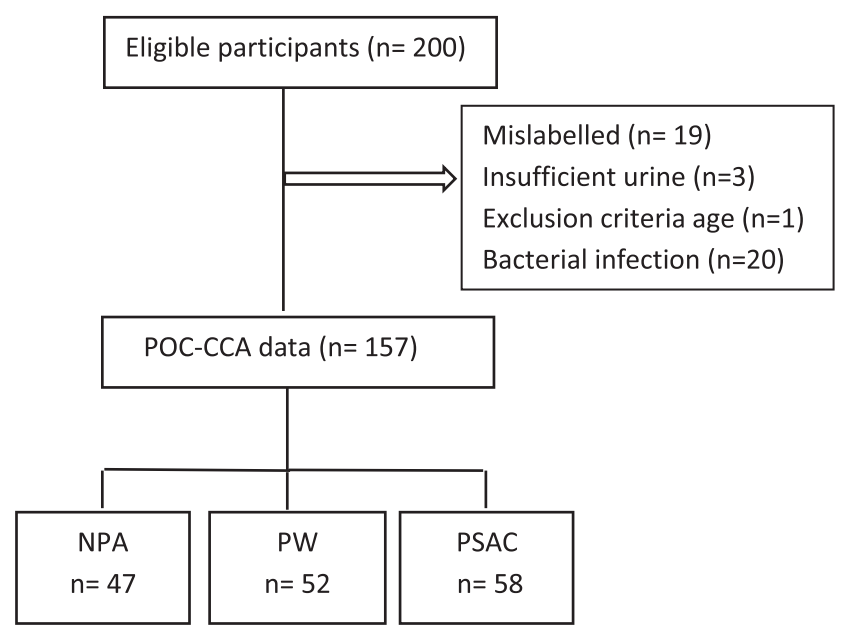

FIGURE 1. Flowchart of study participation. NPAs = nonpregnant adults; $\mathrm{PSAC}=$ preschool-aged children; $\mathrm{PW}=$ pregnant women. 
outcome when traces of the POC-CCA strip test were considered positive and five (11\%) when traces were considered negative. In the 52 PW urines, 29 (56\%) showed a positive POC-CCA result when traces were considered positive and 17 (33\%) when traces were considered negative. Finally, of the 58 tested PSAC, 43 (74\%) had a positive result when traces were considered positive and 27 (47\%) when traces were considered negative (Table 1). These findings resulted in a specificity of the POC-CCA test in PW and PSAC of $67 \%$ and $53 \%$, respectively, compared with $89 \%$ in NPAs when excluding traces.

Overall, PSAC showed significantly higher visual scores (median score of 0.5; IQR: 0-2) than PW (median score of 0.5; IQR: 0-1) and NPAs (median score of 0; IQR: 0-2) (Figure 2).

Correlation between the POC-CCA visual score and age and/or pregnancy trimester of the participants per group is shown in Figure 3. In the NPA group (Figure 3A and B), 26 males and 21 females in the age range of 20-62 years were tested, of which three females scored $3+$ and one female scored $2+$, whereas only one male scored $2+$. All five were aged between 21 and 30 years. In the PW group (Figure $3 \mathrm{C}$ and D) the pregnancy trimester and the age of the 52 included women are shown. Pregnant women from all trimesters showed positive scores with a trend toward higher positivity (taking traces as positive) at the end of the pregnancy, but no statistically significant association, neither with trimester nor age, was observed. In the PSAC group (Figure 3E and F), the highest visual scores were seen in the newborn babies, with an infant of 9 months of age being the oldest positive, with a score of $2+$. The youngest age-groups ( $<1$ and 1-3 months) were all positive if traces were considered as positive.

Urine reagent strip and UCP-LF CAA. All urines collected from NPAs and PW, as well as 26 urines of 58 PSAC had enough volume to be tested with the UCP-LF CAA test to affirm the absence of schistosome infections in these nonexposed study participants. All individuals who tested positive by POC-CCA were negative in the UCP-LF CAA test.

To dissect underlying mechanism of false-positive POCCCA tests, we tested urines for protein content and $\mathrm{pH}$ by

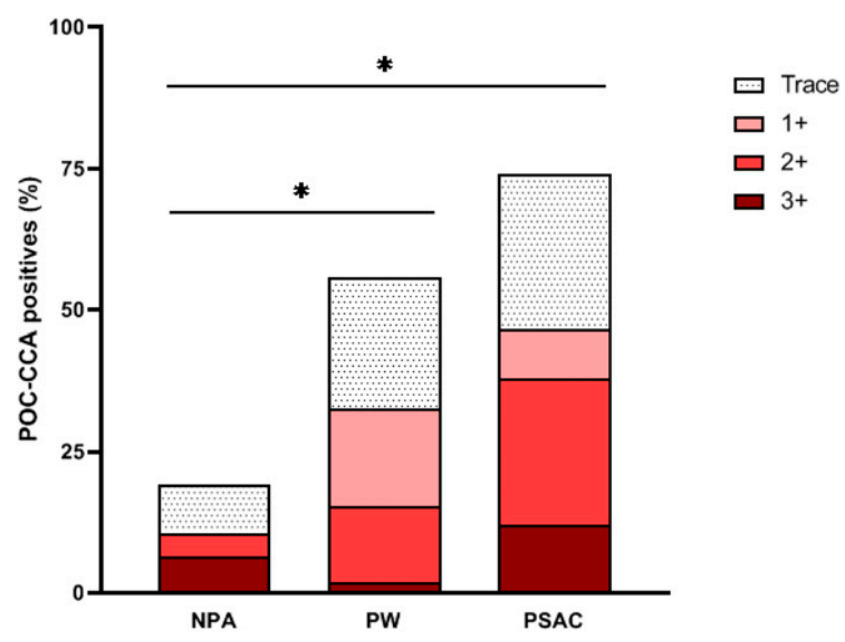

FIGURE 2. Percentage of total positives for POC-CCA (trace, 1+,2+, and $3+)$ for all the three study groups. NPAs $=$ nonpregnant adults $(n=$ 47); POC-CCA = point-of-care-circulating cathodic antigen; $P S A C=$ preschool-aged children $(n=58)$; $\mathrm{PW}=$ pregnant women $(n=52)$. *Indicates significant differences $(P<0.05)$ between the indicated groups and their number of positives (traces not included) based on POC-CCA. This figure appears in color at www.ajtmh.org.

urine reagent strip. Remarkably, 49 urines that score positive for POC-CCA had a lower average ( $\mathrm{pH}$ of 5.9) than the negatives, whereas a total of 108 negative urines that score negative or trace for POC-CCA had an average $\mathrm{pH}$ of 6.9. When analyzed per category, the 5, 17, and 27 urines which scored positive for the POC-CCA test had a pH average of 5.7 (NPAs), 6.3 (PW), and 5.8 (PSAC). Altogether, we observed that all 11 urines which scored +3 for $\mathrm{POC}-\mathrm{CCA}$ had an acidic $\mathrm{pH}$ (below 5.5). Twenty-one urines tested positive for protein, but there was no significant relation between protein and POC-CCA positivity.

To further investigate the association with low $\mathrm{pH}$, nine urines positive by POC-CCA were retested by the same POC-CCA batch with the addition of a drop of neutralizing buffer ( $\mathrm{pH}$ 9; present in previous batches available by Rapid

TABLE 1

Demographic characteristics of the study participants and outcome of the POC-CCA urine test when traces were considered either positive (tr+) or negative (tr-)

\begin{tabular}{|c|c|c|c|c|c|c|c|}
\hline \multirow[b]{2}{*}{ Group } & \multirow[b]{2}{*}{ Gender } & \multirow[b]{2}{*}{ Age-group } & \multirow[b]{2}{*}{ Total tested } & \multicolumn{4}{|c|}{ POC-CCA } \\
\hline & & & & $\mathrm{POS}(\mathrm{tr}+), n(\%)$ & $\mathrm{POS}(\mathrm{tr}-), n(\%)$ & Spec $(\operatorname{tr}+)(\%)$ & Spec (tr-) (\%) \\
\hline \multirow[t]{6}{*}{ Nonpregnant adults } & Females $($ total $=21$ ) & 20-30 years & 16 & $5(31.3)$ & $4(25)$ & 76 & 81 \\
\hline & & $31-34$ years & 2 & 0 & 0 & & \\
\hline & & $35-62$ years & 3 & 0 & 0 & & \\
\hline & Males $($ total $=26)$ & $20-30$ years & 17 & $3(17.6)$ & $1(5.8)$ & 85 & 96 \\
\hline & & $31-34$ years & 3 & 1 (33.3) & 0 & & \\
\hline & & $35-62$ years & 6 & 0 & 0 & & \\
\hline \multirow[t]{3}{*}{ Pregnant women } & Females $($ total $=52)$ & $20-30$ years & 17 & $11(61.1)$ & $6(33.3)$ & 44 & 67 \\
\hline & & $31-34$ years & 19 & $11(57.9)$ & $6(31.6)$ & & \\
\hline & & $35-46$ years & 16 & 7 (43.8) & 5 (31.3) & & \\
\hline \multirow[t]{8}{*}{ Preschool-aged children } & Females (total $=23$ ) & 0 month & 7 & $7(100)$ & $6(85.7)$ & 22 & 61 \\
\hline & & 1-3 months & 4 & $4(100)$ & $2(50)$ & & \\
\hline & & 4-12 months & 5 & $3(60)$ & $1(20)$ & & \\
\hline & & 13-47 months & 7 & $4(57.1)$ & 0 & & \\
\hline & Males $($ total $=35)$ & 0 month & 8 & $8(100)$ & $6(75)$ & 29 & 49 \\
\hline & & 1-3 months & 10 & $10(100)$ & $8(80)$ & & \\
\hline & & 4-12 months & 10 & $6(60)$ & $4(40)$ & & \\
\hline & & 13-47 months & 7 & $1(14.3)$ & 0 & & \\
\hline
\end{tabular}


A

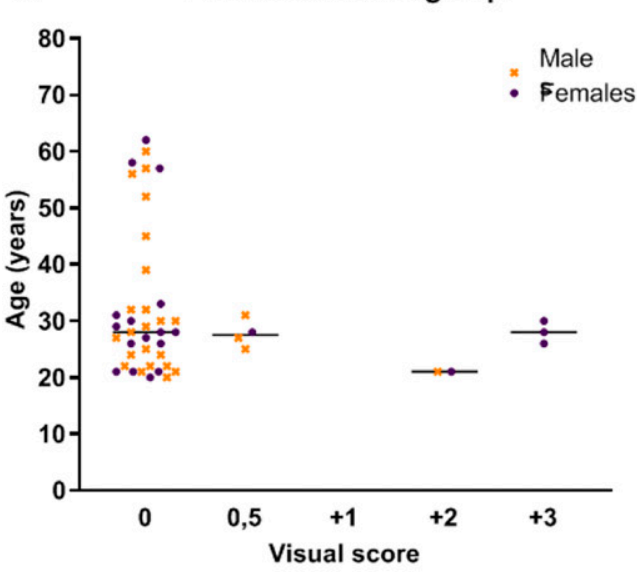

C

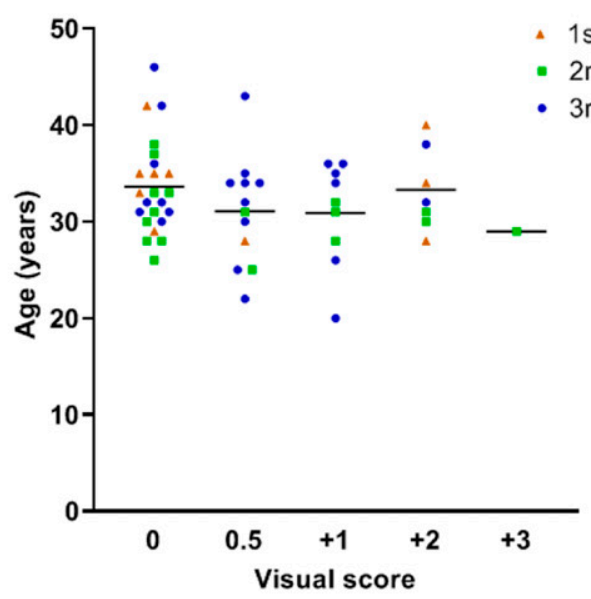

E POC-CCA in PSAC group

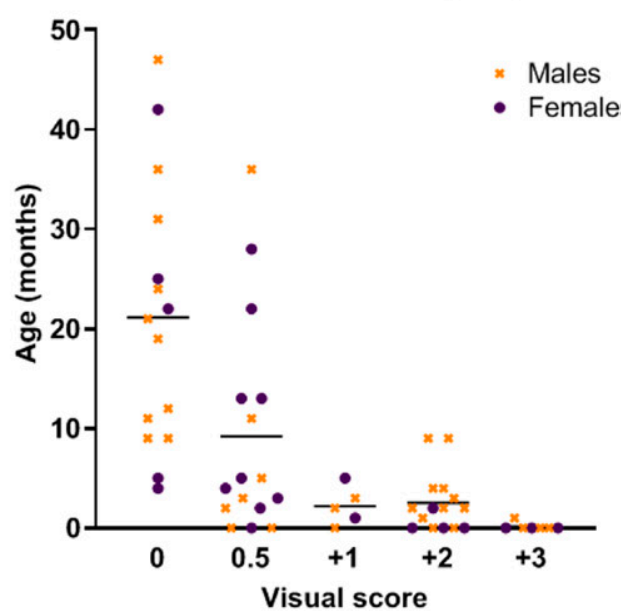

B

POC-CCA in NPA per gender

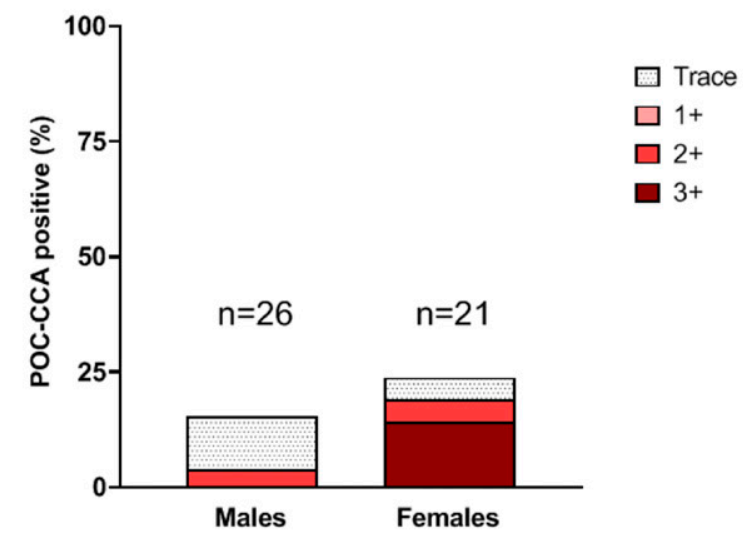

D POC-CCA in PW per trimester

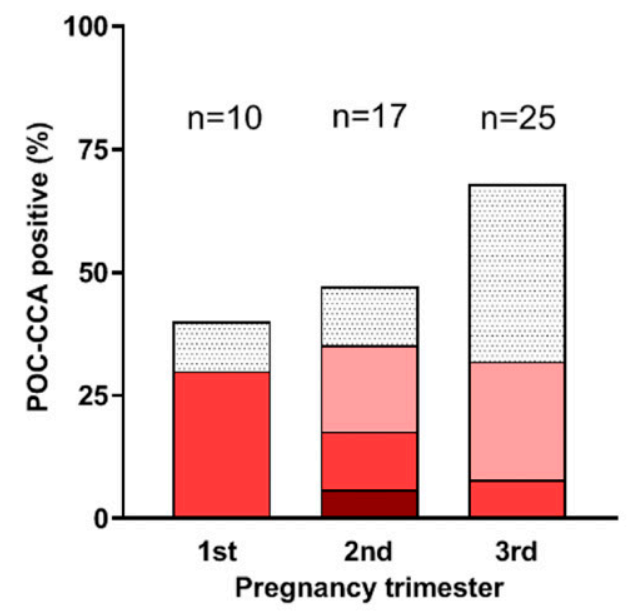

F POC-CCA in PSAC per age groups

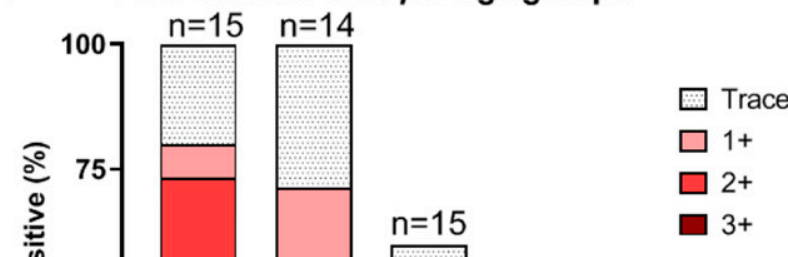

FIGURE 3. Scatterplot by visual score (negative, trace [0.5], 1+,2+, and 3+) for NPAs based on gender, age, and percentage of total positives (A and $\mathbf{B})$. For PW based on pregnancy trimester, age and percentage of total positives (C and $\mathbf{D}$ ) and for PSAC based on gender, age, and percentage of total positives $(\mathbf{E}$ and $\mathbf{F})$. NPAs = nonpregnant adults; PSAC = preschool-aged children; PW = pregnant women. This figure appears in color at www.ajtmh.org.

Medical Diagnostics) that was added to the cassette after dispensing the drop of urine. For direct comparison, the same urine sample as used previously was retested. All urine samples turned negative after addition of the buffer, whereas only one changed into a trace. Samples were also tested with an additional POC-CCA batch to rule out batch-to-batch variation of the false positivity from these groups, with similar results (data not shown). 


\section{DISCUSSION}

Urines from PW and children younger than 4 years living in the Netherlands with no Schistosoma infection were tested for POC-CCA and compared with nonpregnant, healthy adults' urines. This study found high numbers of false-positive results, in particular in the urines of PW and infants.

The POC-CCA test has been very successfully applied for the detection of $S$. mansoni infections in areas in subSaharan Africa highly endemic for schistosomiasis. ${ }^{5,6,23,24}$ In addition, the assay has been previously reported to give positive results in urine of uninfected $\mathrm{PW}$, which is most likely due to sharing of LeX trisaccharide-containing epitopes between excreted host glycans or glycoconjugates and the repeating units of LeX that form the immunoreactive part of CCA. ${ }^{10,18}$ The LeX carbohydrate motif is found on a subset of human cells as well as on a number of secreted glycoproteins and may therefore be a cause of cross-reactivity with CCA or other nonhost-derived antigens that present this carbohydrate motif during infections. ${ }^{10}$ In several occasions, it has been suggested that increased background in POC-CCA is associated with epitopes similar to CCA-associated LeX produced in urinary tract infections, tumors, hematuria, and pregnancy (e.g., stage-specific embryonic antigen-1, CD15, or carcinoembryogenic antigen), thus interfering with the result interpretation. ${ }^{15,18,25,26}$ Possibly highly glycosylated proteins found in urine that are under the regulation of pregnancy-related hormones, for example, Tamm-Horsfall glycoprotein (Uromodulin) and Glycodelin-also contain CCA cross-reactive glycans, which could explain the observations of pregnancy-related false positivity. ${ }^{27}$ This component might be a glycoprotein as, for example, human chorionic gonadotropin which is produced in high concentrations during pregnancy in increasing amounts and which also has been reported to have cross-reactive epitopes with other hormones. ${ }^{28,29}$ However, in this study, we only observed a nonsignificant trend between POC-CCA score and pregnancy trimester.

Moreover, glycans, for example, the LeX-containing LNFPIII pentasaccharide and various glycosylated proteins, are also found in human breast milk, which may contribute to the presence of CCA cross-reactivity in PW as well as young breast milk-fed infants. Pregnant women in later stages of pregnancy are more likely to get bacterial infections, and such conditions cannot be discarded to influence the POC-CCA result. $^{30}$ Other hypotheses could include microscopic hematuria in pregnancy and preeclampsia (disorder of pregnancy characterized by the onset of high blood pressure and often a significant amount of protein in the urine). Although these factors could in theory add background to the POC-CCA test, we found in our study only one PW that had protein presence, and this urine was negative for POC-CCA. Erythrocytes and hemoglobin were not found in any of the PW urines; thus, we can discard a possible background based on the aforementioned parameters for this group. At the current stage, it remains unclear which component(s) exactly is (are) the cause of the false positivity, and focused further studies are needed to resolve this.

In this study, a total of 27 PSAC (46.5\%) scored positive for POC-CCA, and all of them were babies up to 9 months old with a clear trend toward newborns. As lower POC-CCA scores were associated with older infants, this may suggest that the interfering molecule could be associated with age-related aspects such as nutritional components, via mother-umbilical cord or placenta-or, after birth, via breastfeeding.

A total of 17 (32.7\%) PW were positive for POC-CCA, indicating a possible cross-reaction with an interfering component in the urine potentially different from PSAC. Results from $\mathrm{pH}$ testing suggest that the acidity of the urines is leading to false positivity in the POC-CCA test. This indeed seems to be the case as the positive reactions were removed after addition of a neutralizing buffer. A low pH could cause several alterations in the monoclonal antibody-CCA interaction as well as to the protein binding to the nitrocellulose and thus affect test performance. Also, by a simple a priori sample treatment such as TCA, the false positivity could be inhibited, but then a neutralization step should be included in the suggested procedure.

This study shows that false-positive reactions may appear in POC-CCA testing, particularly in some specific population groups. The test design, in combination with the presence of cross-reactive urinary glycans or glycoconjugates, appears to be the cause of the observed false-positive results. Previously, Colley et al. ${ }^{5}$ showed a very high specificity of $99 \%$ for the POC-CCA in children from a non-endemic Schistosoma area, using the previous POC-CCA format in which a drop of buffer was added on the sample pad after adding the urine sample. This suggests that the specificity of the current test can be significantly improved by reintroducing the buffer addition step. Future QC checks of the POC-CCA tests should always include urines collected from different age-groups and gender, in particular if the POC-CCA test is meant to be used outside school programs. ${ }^{5}$

Concluding, the POC-CCA test appears to have a lower specificity in young infants (younger than 9 months), as well as in PW, but this may be improved by modifications in the buffers used in the test. Further investigations in individuals from endemic areas, in menstruating women, linking data from mother-child pairs, and the influence of breastfeeding, should give more insight into this phenomenon. It would also be interesting to identify the molecules causing the cross-reactivity and consequently false positivity, which may help us to find a technical solution of the problem. Overall, as the general use of the POC-CCA will not be for the groups of individuals studied here, it remains a user-friendly, noninvasive, and sensitive test providing a good estimate of the prevalence and intensity of Schistosoma mansoni in endemic areas.

Received September 9, 2020. Accepted for publication November 25, 2020.

Published online February 1, 2021.

Acknowledgments: We greatly thank all the participants for their participation to this study and the gynecology department and the clinical chemical laboratory of the LUMC for their collaboration.

Authors' addresses: Miriam Casacuberta-Partal, Margreet Beenakker, Pytsje T. Hoekstra, Lisa Kroon, Dieuwke Kornelis, Cornelis H. Hokke, Govert J. van Dam, and Lisette van Lieshout, Department of Parasitology, Leiden University Medical Centre, Leiden, The Netherlands, E-mails: m.casacuberta_partal@lumc.nl, margreetbeenakker@ gmail.com, p.t.hoekstra-mevius@lumc.nl, lisajkroon@gmail.com, d.kornelis@lumc.nl, c.h.hokke@lumc.nl, g.j.van_dam@lumc.nl, and e.a.van_lieshout@lumc.nl. Claudia J. de Dood and Paul L. A. M Corstjens, Department of Cell and Chemical Biology, Leiden University Medical Center, Leiden, The Netherlands, E-mails: c.j.de_dood@ lumc.nl and p.l.a.m.corstjens@lumc.nl. Meta Roestenberg, Department of Parasitology, Leiden University Medical Centre, Leiden, The Netherlands, and Department of Infectious Diseases, Leiden 
University Medical Centre, Leiden, The Netherlands, E-mail: m.roestenberg@lumc.nl.

\section{REFERENCES}

1. Colley DG, Bustinduy AL, Secor WE, King CH, 2014. Human schistosomiasis. Lancet 383: 2253-2264.

2. World Health Organization, 2020. Schistosomiasis. Geneva, Switzerland: WHO. Available at: https://www.who.int/en/newsroom/fact-sheets/detail/schistosomiasis. Accessed June 22, 2020.

3. Utzinger J, Becker SL, van Lieshout L, van Dam GJ, Knopp S, 2015. New diagnostic tools in schistosomiasis. Clin Microbiol Infect 21: 529-542.

4. van Dam GJ, Wichers JH, Ferreira TM, Ghati D, van Amerongen A, Deelder AM, 2004. Diagnosis of schistosomiasis by reagent strip test for detection of circulating cathodic antigen. $J$ Clin Microbiol 42: 5458-5461.

5. Colley DG et al., 2013. A five-country evaluation of a point-of-care circulating cathodic antigen urine assay for the prevalence of Schistosoma mansoni. Am J Trop Med Hyg 88: 426-432.

6. Colley DG et al., 2020. Contributions of the Schistosomiasis Consortium for Operational Research and Evaluation (SCORE) to schistosomiasis control and elimination: key findings and messages for future goals, thresholds, and operational research. Am J Trop Med Hyg 103 (Suppl 1): 125-134.

7. Clements $M N$ et al., 2018. Latent class analysis to evaluate performance of point-of-care CCA for low-intensity Schistosoma mansoni infections in Burundi. Parasit Vectors 11: 111.

8. Barenbold $\mathrm{O}$ et al., 2018. Translating preventive chemotherapy prevalence thresholds for Schistosoma mansoni from the KatoKatz technique into the point-of-care circulating cathodic antigen diagnostic test. PLoS Negl Trop Dis 12: e0006941.

9. World Health Organization, 2018. Enhancing Implementation of Schistosomiasis Control and Elimination Programmes. Geneva, Switzerland: WHO. Available at: https://www.who.int/activities/ enhancing-implementation-of-schistosomiasis-control-andelimination-programmes. Accessed August 19, 2020.

10. Van Dam GJ, Bergwerff AA, Thomas-Oates JE, Rotmans JP, Kamerling JP, Vliegenthart JF, Deelder AM, 1994. The immunologically reactive $\mathrm{O}$-linked polysaccharide chains derived from circulating cathodic antigen isolated from the human blood fluke Schistosoma mansoni have Lewis $\mathrm{x}$ as repeating unit. Eur J Biochem 225: 467-482.

11. Casacuberta-Partal M, Hoekstra PT, Kornelis D, van Lieshout $L$, van Dam GJ, 2019. An innovative and user-friendly scoring system for standardised quantitative interpretation of the urinebased point-of-care strip test (POC-CCA) for the diagnosis of intestinal schistosomiasis: a proof-of-concept study. Acta Trop 199: 105150.

12. Lamberton PH, Kabatereine NB, Oguttu DW, Fenwick A, Webster JP, 2014. Sensitivity and specificity of multiple Kato-Katz thick smears and a circulating cathodic antigen test for Schistosoma mansoni diagnosis pre- and post-repeated-praziquantel treatment. PLoS Negl Trop Dis 8: e3139.

13. Prada JM, Touloupou P, Adriko M, Tukahebwa EM, Lamberton PHL, Hollingsworth TD, 2018. Understanding the relationship between egg- and antigen-based diagnostics of Schistosoma mansoni infection pre- and post-treatment in Uganda. Parasit Vectors 11: 21

14. Coulibaly JT, N'Gbesso YK, Knopp S, N'Guessan NA, Silue KD, van Dam GJ, N'Goran EK, Utzinger J, 2013. Accuracy of urine circulating cathodic antigen test for the diagnosis of Schistosoma mansoni in preschool-aged children before and after treatment. PLoS Negl Trop Dis 7: e2109.

15. Kildemoes AO, Vennervald BJ, Tukahebwa EM, Kabatereine NB, Magnussen P, de Dood CJ, Deelder AM, Wilson S, van Dam GJ,
2017. Rapid clearance of Schistosoma mansoni circulating cathodic antigen after treatment shown by urine strip tests in a Ugandan fishing community - relevance for monitoring treatment efficacy and re-infection. PLoS Negl Trop Dis 11: e0006054.

16. Bustinduy AL, Stothard JR, Friedman JF, 2017. Paediatric and maternal schistosomiasis: shifting the paradigms. Br Med Bull 123: 115-125.

17. Stothard JR et al., 2011. Closing the praziquantel treatment gap: new steps in epidemiological monitoring and control of schistosomiasis in African infants and preschool-aged children. Parasitology 138: 1593-1606.

18. Greter H, Krauth SJ, Ngandolo BN, Alfaroukh IO, Zinsstag J, Utzinger J, 2016. Validation of a point-of-care circulating cathodic antigen urine cassette test for Schistosoma mansoni diagnosis in the sahel, and potential cross-reaction in pregnancy. Am J Trop Med Hyg 94: 361-364.

19. Rapid Medical Diagnostics, 2018. Rapid Test for Qualitative Detection of: Bilharzia (Schistosomiasis). Available at: https://www.rapid-diagnostics.com/updates_15_09_2019/ RMD_Pamphlet_13_12_2018_Colourweb.pdf. Accessed June 22, 2020.

20. Machin D, Campbell MJ, Tan SB, Tan SH, 2011. Sample Size Tables for Clinical Studies. Chichester, United Kingdom: John Wiley \& Sons, 38-40.

21. Corstjens $P$ et al., 2020. Circulating anodic antigen (CAA): a highly sensitive diagnostic biomarker to detect active schistosoma infections-improvement and use during SCORE. Am J Trop Med Hyg 103 (Suppl 1): 50-57.

22. Corstjens PL, Nyakundi RK, de Dood CJ, Kariuki TM, Ochola EA, Karanja DM, Mwinzi PN, van Dam GJ, 2015. Improved sensitivity of the urine CAA lateral-flow assay for diagnosing active Schistosoma infections by using larger sample volumes. Parasit Vectors 8: 241.

23. Coulibaly JT et al., 2011. Accuracy of urine circulating cathodic antigen (CCA) test for Schistosoma mansoni diagnosis in different settings of Cote d'Ivoire. PLoS Negl Trop Dis 5: e1384.

24. Tchuem Tchuente LA, Kuete Fouodo CJ, Kamwa Ngassam RI, Sumo L, Dongmo Noumedem C, Kenfack CM, Gipwe NF, Nana ED, Stothard JR, Rollinson D, 2012. Evaluation of circulating cathodic antigen (CCA) urine-tests for diagnosis of Schistosoma mansoni infection in Cameroon. PLoS Negl Trop Dis 6: e1758.

25. Colley DG, Andros TS, Campbell CH, Jr., 2017. Schistosomiasis is more prevalent than previously thought: what does it mean for public health goals, policies, strategies, guidelines and intervention programs? Infect Dis Poverty 6: 63.

26. Thomas PG, Harn DA, Jr., 2004. Immune biasing by helminth glycans. Cell Microbiol 6: 13-22.

27. Seppala M, Taylor RN, Koistinen H, Koistinen R, Milgrom E, 2002. Glycodelin: a major lipocalin protein of the reproductive axis with diverse actions in cell recognition and differentiation. Endocr Rev 23: 401-430.

28. Birken S, Gawinowicz MA, Kardana A, Cole LA, 1991. The heterogeneity of human chorionic gonadotropin (hCG). II. Characteristics and origins of nicks in hCG reference standards. Endocrinology 129: 1551-1558.

29. Porakishvili N, Chiesa MD, Chikadze N, Martensen P, Justesen J, Lund T, Delves PJ, Roitt IM, 2002. Elimination of luteinizing hormone cross-reactive epitopes from human chorionic gonadotropin. Vaccine 20: 2053-2059.

30. Kalinderi K, Delkos D, Kalinderis M, Athanasiadis A, Kalogiannidis I, 2018. Urinary tract infection during pregnancy: current concepts on a common multifaceted problem. J Obstet Gynaecol 38: 448-453. 